\title{
BMJ Open Exploring clinicians' perspectives on the 'Obstetric Anal Sphincter Injury Care Bundle' national quality improvement programme: a qualitative study
}

\author{
Posy Bidwell (D , , ${ }^{1}$ Ranee Thakar, ${ }^{2}$ Ipek Gurol-Urganci (D) , ${ }^{3}$ James M Harris, ${ }^{4}$ \\ Louise Silverton, ${ }^{5}$ Alexandra Hellyer, ${ }^{1}$ Robert Freeman, ${ }^{6}$ Edward Morris, ${ }^{1}$ \\ Vivienne Novis, ${ }^{1}$ Nick Sevdalis (1) ${ }^{7}$
}

To cite: Bidwell $P$, Thakar $\mathrm{R}$, Gurol-Urganci I, et al. Exploring clinicians' perspectives on the 'Obstetric Anal Sphincter Injury Care Bundle' national quality improvement programme: a qualitative study. BMJ Open 2020;10:e035674. doi:10.1136/ bmjopen-2019-035674

- Prepublication history and additional material for this paper are available online. To view these files, please visit the journal online (http://dx.doi. org/10.1136/bmjopen-2019035674).

Received 11 November 2019 Revised 07 May 2020

Accepted 30 June 2020

Check for updates

(C) Author(s) (or their employer(s)) 2020. Re-use permitted under CC BY-NC. No commercial re-use. See rights and permissions. Published by BMJ.

For numbered affiliations see end of article.

Correspondence to

Dr Posy Bidwell;

pbidwell@rcog.org.uk

\section{ABSTRACT}

Introduction Obstetric anal sphincter injuries (OASI) can have severe debilitating consequences to women and health systems. The OASI Care Bundle quality improvement programme was introduced in 16 maternity units across England, Scotland and Wales (January 2017 to March 2018) to address increasing OASI rates.

Objectives To explore clinicians' (midwives' and obstetricians') perspectives of the OASI Care Bundle with respect to (1) acceptability, (2) feasibility, and (3) sustainability.

Design A qualitative exploratory study using focus groups methodology.

Setting A total of 16 focus groups were conducted in 16 maternity units in England, Scotland and Wales where the OASI Care Bundle was implemented. Focus groups took place approximately 3 months following initial implementation of the care bundle in each unit. Participants A total of 101 clinicians participated, with an average of six per focus group. Participants volunteered to take part and compromised of 37 obstetricians and 64 midwives (including eight students). The majority were female and the mean age was 36.5 years.

Results Four main themes emerged: 'Implementation strategies', 'Opportunities to use the OASI Care Bundle', 'Does current practice need to change?' and 'Perceptions of what women want'. Midwives were more likely than obstetricians to report themes alluding to 'what women want' and variations in intrapartum perineal protection techniques. Both professional groups reported similar views of other themes, in particular regarding the supporting clinical evidence. Gaps were identified in clinicians' knowledge and experience of intrapartum perineal management.

Conclusions Adoption of the OASI Care Bundle was associated with a number of cognitive and interpersonal factors, such as personal values, interprofessional working and how the intervention was launched; which both facilitated and impeded adoption. The 'what women want' theme has implications for maternal autonomy and needs further exploration. Our findings can be used by similar initiatives to reduce perineal trauma both nationally and internationally.

Trial registration number ISCTRN 12143325; https://doi. org/10.1186/ISRCTN12143325.
Strengths and limitations of this study

- This study explores the implementation of the Obstetric Anal Sphincter Injury (OASI) Care Bundle and highlights the importance of how interpersonal and cognitive factors affected adoption.

- Focus groups were conducted in 16 UK maternity units that implemented the OASI Care Bundle-the wide coverage and qualitative methodology provide deep insights into the barriers and enablers of improvement.

- Study generalisability may be limited due to participant's self-selection to attend.

- The findings of this study provide a useful blueprint for the implementation of improvement interventions throughout maternity services.

\section{BACKGROUND}

An obstetric anal sphincter injury (OASI) refers to any injury to the anal sphincter muscle sustained during childbirth. Graded as third or fourth-degree tears, depending on severity, they can cause significant long-term morbidities including anal incontinence and post-traumatic stress disorder. ${ }^{1}$ These complications can severely impact a woman's quality of life and affect future birth choices. ${ }^{23}$ There are also significant long-term financial consequences for health systems associated with further treatment and litigation claims-in the UK these were an estimated $£ 31.2$ million between 2000 and 2010, making it the fourth largest number of maternity claims. ${ }^{4}$

There are known demographic and intrapartum risk factors for OASI, ${ }^{5}$ but for some women there is no clear reason why they sustain these injuries. Identified contributing factors include lack of training, lack of awareness and variation with intrapartum practiceparticularly with regard to a 'hands-poised' or 'hands-off' approach to the perineum. ${ }^{6-9}$ 


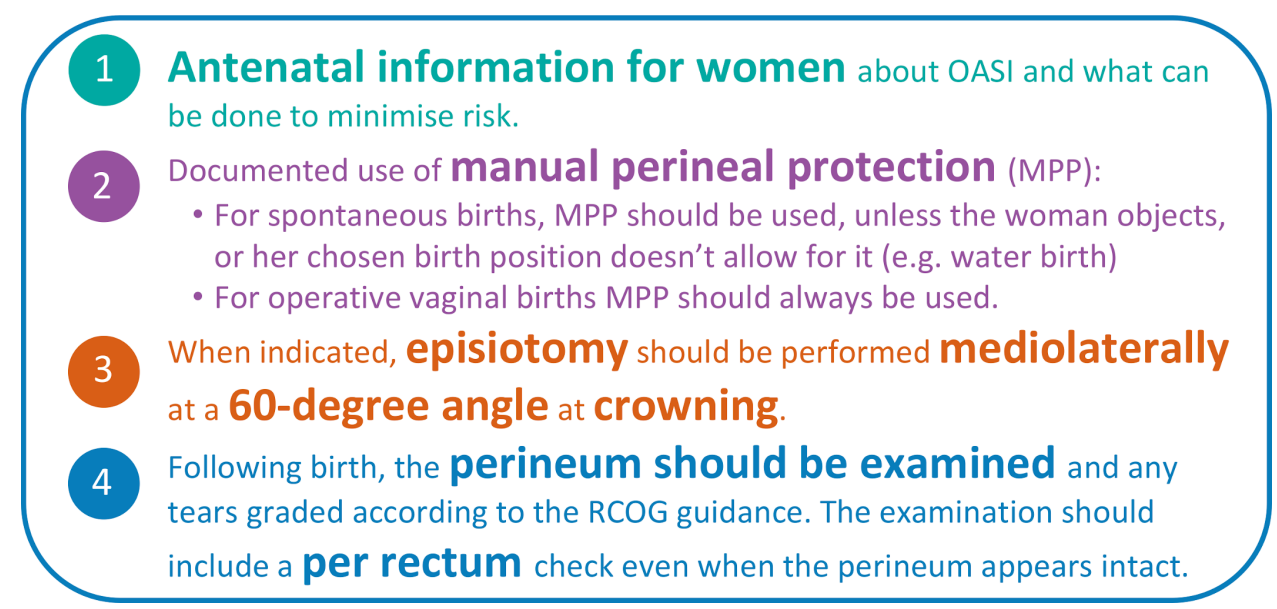

Figure 1 The four discrete elements of the OASI Care Bundle. OASI, obstetric anal sphincter injury; RCOG, Royal College of Obstetricians and Gynaecologists.

Adoption of interventions using evidence-based practice and increased awareness significantly reduced rates in Scandinavian countries ${ }^{10-12}$ and in small-scale studies in England. $^{1314}$

However, OASI rates continue to increase: in the UK, they tripled among primiparous women over a 10-year period. ${ }^{15}$ Similar rising trends have been reported in several countries including Australia, ${ }^{16}$ Canada $^{5}$ and China. ${ }^{17}$ Clinical improvement to reduce this pattern is required.

This clinical need provided a strong driver to implement the 'OASI Care Bundle' quality improvement (QI) programme at national level to attempt to reverse this trend in the UK. The programme and evaluation background, design and methods have been reported in detail. ${ }^{18}$ Briefly, the QI programme involved an intervention comprising a care bundle, an awareness campaign and multidisciplinary training implemented across $16 \mathrm{UK}$ maternity units (January 2017 to April 2018). Figure 1 shows the elements of the OASI Care Bundle. Local implementation of the care bundle was facilitated by obstetric and midwifery champions within participating units.

Implementation of the care bundle reduced OASI rates from $3.3 \%$ to $3.0 \% \quad(p=0.03)$, with over 55000 women included in the analysis. ${ }^{19}$ In addition to assessing the clinical effectiveness of the intervention, the evaluation sought to understand the feasibility and utility of the implementation strategies of the bundle across units. The aim of this paper is to report a detailed exploration of clinicians' (midwives' and obstetricians') perspectives of the care bundle implementation and adoption within their units.

\section{METHODS}

\section{Study design and methodology}

Focus groups (FG) were conducted with clinicians (obstetricians and midwives) across all 16 participating units to explore the acceptability and feasibility of the OASI Care Bundle. A standard set of questions were used (see online supplementary material 1) as a basis of semistructured FGs to explore the intervention (the care bundle, the local training and the awareness campaign), the implementation strategy and the local context within each unit.

\section{Recruitment}

Sixteen FGs were conducted between March and December 2017-one at each maternity unit in order to ensure representation from all participating units and give them all an opportunity to give their views of the bundle. In doing so, we expected data saturation to be reached after six to eight FGs and indeed reached saturation after eight FGs. The FGs were scheduled to take place 6-8 weeks after the start of implementation; however, for logistical reasons, in some units, this extended up to 12 weeks. Local champions at each unit assisted with recruitment by advertising (via posters, email and face-to-face discussions), when the FG would take place and by highlighting that the discussion was an opportunity for clinicians to provide their views of the care bundle.

The aim was for each FG to reflect a range of age and experience; however, due to staffing levels and workload within each unit a pragmatic approach was taken to recruitment. Using convenience sampling, eligible participants were obstetricians and midwives (including student midwives) who were working in each of the 16 maternity units. Obstetricians and midwives who were interested in taking part came to the FG in their unit, where the moderator (PB) provided more information about the study. Those who consented then took part in the discussion.

The recommendation is that FGs comprise between four and eight participants ${ }^{20}$; however, as study participants typically comprised clinicians who were on shift at the time of the FG, one FG had less $(n=3)$ and one had more $(n=10)$ than this recommended number. The average number of participants was 6 . Table 1 shows the FG composition across the four study regions. 
Table 1 Focus group composition across study regions

\begin{tabular}{|c|c|c|c|c|c|c|}
\hline Unit No & Type of unit & Size of unit* & Midwives & Student midwives & Obstetricians & Total \\
\hline \multirow[t]{2}{*}{ Region 1} & $2 \mathrm{OU}+\mathrm{AMU}$ & 1 small & 14 & 1 & 17 & 32 \\
\hline & $1 \mathrm{OU}+\mathrm{FMU}$ & 2 medium & & & & \\
\hline \multirow[t]{2}{*}{ Region 2} & $1 \mathrm{OU}$ & & 14 & 3 & 4 & 21 \\
\hline & $2 \mathrm{OU}+\mathrm{AMU}$ & 2 medium & & & & \\
\hline \multirow[t]{3}{*}{ Region 3} & $4 \mathrm{OU}+\mathrm{AMU}$ & 2 small & 13 & 2 & 8 & 23 \\
\hline & & 1 medium & & & & \\
\hline & & 1 large & & & & \\
\hline Region 4 & $2 \mathrm{OU}$ & $\begin{array}{l}1 \text { small } \\
1 \text { medium }\end{array}$ & 16 & 2 & 8 & 26 \\
\hline
\end{tabular}

${ }^{*}$ Categorised according to number of births per year: small $<3500$, medium 3500-5000, large $>5000$.

$\mathrm{AMU}$, alongside midwifery unit; FMU, free-standing midwifery unit; $\mathrm{OU}$, obstetric unit.

\section{FG conduct}

FGs took place in meeting rooms within the maternity units. Sessions lasted on average 30 minutes (range 23-49 minutes). Before the FG began, participants were asked to complete a questionnaire to obtain basic, non-identifiable, demographic information (see online supplementary material 2).

\section{Patient and public involvement}

The OASI Project had patient and public involvement (PPI) throughout inception, implementation and evaluation stages. The project was supported by an independent advisory group, including lay representatives. The antenatal information sheet (first component of the OASI Care Bundle) was developed together with PPI groups in order to ensure that the material was appropriate.

\section{Analysis}

All 16 FGs were moderated by the same person (PB). As a midwife, the moderator (PB) had a good understanding of the topic and as a qualitative experienced researcher was able to build a productive and trusting relationship with the participants, none of whom were previously known to her. Participants were informed of the moderator's credentials and profession during the introduction. Participants gave their consent for the discussion to be audio recorded. All audio recordings were transcribed verbatim for analysis. All transcripts were anonymised, with no personal identifiable markers. Analysis was based on the transcripts and moderator's reflections from the field (ie, a form of autoethnography).

Grounded theory was used as it allows for emerging themes to be developed using an iterative process. ${ }^{21}$ Data were analysed inductively as we were interested in understanding new information and insights. ${ }^{22}$ NVivo V.11 facilitated data analysis. Transcripts were read and re-read several times and coded by the researcher (PB). 'Axial coding' then determined causal or consequential relationships between the codes to identify dominant themes. An important part of grounded theory is the ability to test concepts with colleagues who have experience in the area. $^{21}$ To ensure such rigour, an additional researcher (JMH) reviewed the codes and themes to develop new insights and minimise bias. For a theme to be confirmed it had to be indicated by the data on several occasions. This process indicated data saturation, meaning that no new themes would have been identified with the inclusion of further data. The collection and analysis of data adhered to the Standards for Reporting Qualitative Research. ${ }^{23}$

\section{RESULTS}

In total, 101 clinicians participated, comprising 37 obstetricians, 56 midwives and 8 student midwives. The characteristics of participants are presented in table 2.

Four major themes were identified which describe the clinicians' perspectives on the implementation of the care bundle. Within these four themes, there were several subthemes. These are outlined in figure 2 and described in full below.

\section{Theme 1: Implementation strategies}

This theme reflected the way with which the care bundle was introduced and implemented within participating units. There were three subthemes, namely: (1) no consultation about the change, (2) introduction of the OASI Care Bundle, and (3) training approaches.

No consultation about the change

Some participants felt that the wider maternity team on the 'shop floor' had not been consulted about the unit's involvement with the OASI Project and the changes this 


\begin{tabular}{|c|c|c|}
\hline Demographic & $\mathbf{n}$ & $\%$ \\
\hline \multicolumn{3}{|l|}{ Age group (years) } \\
\hline$<30$ & 29 & 28.7 \\
\hline $30-39$ & 37 & 36.6 \\
\hline $40-49$ & 14 & 13.9 \\
\hline$\geq 50$ & 21 & 20.8 \\
\hline \multicolumn{3}{|l|}{ Gender } \\
\hline Female & 95 & 94.1 \\
\hline Male & 6 & 5.9 \\
\hline \multicolumn{3}{|c|}{ Clinical area (midwives only, $n=56$ ) } \\
\hline Labour ward & 41 & 73.2 \\
\hline $\begin{array}{l}\text { Community/birth } \\
\text { centre }\end{array}$ & 13 & 23.2 \\
\hline Other & 2 & 3.6 \\
\hline \multicolumn{3}{|c|}{ Years' experience of maternity } \\
\hline$<3$ & 20 & 19.8 \\
\hline $3-5$ & 17 & 16.8 \\
\hline $6-10$ & 26 & 25.7 \\
\hline$>10$ & 38 & 37.6 \\
\hline
\end{tabular}

would bring. This perceived lack of consultation created some reservations about the intervention. Some felt their autonomy had been compromised and that there should have been a more collaborative approach in the early stages for a sense of ownership, given that for many the intervention required a change in practice.
I'm sorry but I felt that it was implemented at [names unit] without any discussion about what midwives wanted. I felt very strongly, as an autonomous practitioner, if there is a reason to do something I will try and do it....Whilst I'm really willing to learn, for me, it didn't feel like a positive step in the care that I give to women. (Midwife)

Conversely, some participants reported that engagement with the project had been created by pre-implementation discussions about the care bundle and perineal trauma. This increased the enthusiasm and appetite for the introduction of the care bundle. This atmosphere may have been more marked in units who were in the later waves of implementation:

I think we'd been waiting for it and asking when it's coming. It's nice to know that it's here, and hopefully we can see what impact that has on our third degree tear rate. (Obstetrician)

\section{Introduction of the OASI Care Bundle}

Prior to implementation, units were sent promotional materials to raise awareness about the project and the long-term consequences of OASI. The local champions attended a Skills Development Day at the Royal College of Obstetricians and Gynaecologists and were advised to plan a 'Launch Day' for the first week of roll-out. How this was approached seemed to affect the engagement with the care bundle. Many participants spoke about a welladvertised launch, which created a lot of 'noise' and got the project off to a good start:

Figure 2 The four main themes and subthemes that emerged from the qualitative data. OASI, obstetric anal sphincter injury. 
Yes, we had a launch day at the unit....on that day they had many sessions talking about it. We had the models.....they had pictures of how you would give an episiotomy, things like that, to add to this... There was quite a lot of noise about this. (Midwife)

In some units however, participants seemed less aware of a launch day. This led to some confusion as to whether the care bundle had officially started:

There might have been [a launch], but I couldn't say yes, for definite, so if it happened, I wasn't aware of it...this comes back to the launch, that people don't think it's been launched. (Midwife)

\section{Training approaches}

There were diverse experiences in the way training was conducted by the local champions. Some participants expressed unhappiness about the lack of dedicated time for training sessions in order to ensure a standardised approach:

There hasn't been dedicated time set aside to deliver a consistent message and get proper training and it's just been on the job, come in when you can, do it when you can. (Midwife)

At the same time, some participants expressed their dissatisfaction at the 'fixed' nature of training sessions. There was also disappointment expressed at the inability to attend the official session that was facilitated by the clinical leads for the project:

I thought the times that to attend the training were really fixed and actually not really flexible for midwives....I mean, not every midwife's free at half past eight to come. (Midwife)

The majority reported that training was done ad hoc, with participants being trained in groups or one to one. Many talked about the positive and passionate way with which the champions delivered the training. The presence of in-house champions was felt to be key to the success of the project as they were able to create awareness and encourage people to attend training:

She [the champion] was like a hound! .... if you weren't trained and you were on her list, she would hunt you down....She would come in early to catch people on night shifts and stuff......If you have somebody like that who is passionate about the training and gets the training done, then I think that's what makes it better. (Obstetrician)

The initial intention was for training to be cascaded within units using a 'train-the-trainer' approach, thereby alleviating the training burden on the champions. This, however, did not happen, and the champions did all the training within each unit. Lack of practice with the care bundle was cited by participants as the most common reason for this:
You need to actually practice and work it out in your head before you can then go on and teach it. (Midwife)

\section{Theme 2: Opportunities to use the OASI Care Bundle}

This theme reflected operational factors to using the care bundle. Within this theme there were two subthemes which acted as barriers to using the care bundle (presence of student midwives and change takes time) and one which acted as an enabler (interprofessional working).

\section{Presence of student midwives}

For midwives, one of main issues was that many of them worked with student midwives and so did not have the opportunity to use the care bundle. This meant that many midwives felt that they did not get the exposure to the care bundle that they needed:

If you have a student every time you have a delivery sometimes you don't necessarily get the practice you need. (Midwife)

\section{Change takes time}

Despite some lack of opportunities to use the care bundle, participants were philosophical about the process involved with changing practice. The majority expressed their belief that time was an important factor. Many reminisced about their first ever birth and how 'fiddly' this was and that the care bundle, in particular the manual perineal protection (MPP) element, was unlearning old techniques and getting used to new ones:

It breaks the habits of a lifetime, what we've always done.... How do you do that after you've done that way for 20 odd years? It's weird. It's different. (Midwife)

\section{Interprofessional working}

For those who had used the care bundle, many reported that they had help, or had given help doing this-particularly performing MPP during instrumental births as participants reported that as a single operator it could be difficult to manage all the aspects. This required an additional layer of interprofessional working, with MPP being undertaken by midwives for obstetricians:

So, you'd have a midwife supporting the perineal body while you did your instrumental delivery. (Obstetrician)

And more 'senior' registrars performed MPP for trainees:

If it's just me, I would do it [MPP] myself but I've certainly done it for my juniors when I'm supervising deliveries....so that they've got their hands free. (Obstetrician)

This team approach created genuine feelings of partnership between midwives and obstetricians, who felt 
that the OASI Care Bundle was a project which involved everyone:

This is the only project involving all the shop floor people. The other projects are focused on just a few groups. (Midwife)

\section{Theme 3: Does current practice need to change?}

This theme reflected how clinicians felt about their current practice and their acceptance and readiness to learn new techniques. There were four subthemes: (1) research evidence, (2) clinical judgement, (3) comfort with current practice, and (4) the 'ebb and flow' of maternity practices.

\section{Research evidence}

There were conflicting views about the evidence for the care bundle. Perceptions surrounding the clinical evidence for the care bundle were a prominent barrier to uptake for both obstetricians and midwives:

Because it would be nice to have a number needed to treat sort of thing for that. So if you have to do one thousand PRs [per rectal examinations] to pick up one, is all that indignity worth it? (Obstetrician)

Participants also expressed their belief that while there may be evidence for the care bundle they felt it was not applicable for their practice and so did not need to change:

I know that there's evidence that it reduces severe trauma but I'm not sure that the evidence is that it reduces the trauma for my particular practice. (Midwife)

\section{Clinical judgement}

Clinical judgement was an important factor and there was a lot of discussion about the fact that no two births are the same. While some participants believed that a benefit of the care bundle was that it provided a standardised approach to preventing OASI, others felt that it took away from their clinical judgement. Clinical judgement was associated with having autonomy:

I think it's [the care bundle] taking away autonomy from the practitioner....I think it's good to have an option to use it. But I think that to a certain extent it is taking away that professional judgement. (Midwife)

\section{Comfortable with current practice}

This theme was expressed by both midwives and obstetricians and created some resistance to change. Often more senior clinicians (obstetricians and midwives) had established their own style of practice and were comfortable with this, whereas more junior, or doctors in training (known in the UK as senior house officers (SHO)) were more adopting of new techniques:
The SHOs that are more like, 'Yes, that makes sense, we should do that' because they haven't got their own established technique yet. It's the senior regs [sic] that I've heard that find reasons not to do it. (Obstetrician)

Some participants felt that their practice was not dissimilar from that outlined by the care bundle. Many midwives referred to the impact of the Hands On Or Hands Poised (HOOP) trial (a UK trial which looked at the effect of 'hands-on' vs 'hands-poised' on postnatal perineal pain $)^{24}$ :

We were trained before the HOOP trial, so we always did hands-on...it's not such an alien concept....there are lots of us that haven't really, probably, if we're all truthful, haven't really moved away from hands-on. (Midwife)

\section{The 'ebb and flow' of maternity practices}

Participants felt that certain practices came in and out of fashion. As for the previous theme, reference was made to how 'post HOOP trial' practice had changed from a 'hands-on' to a 'hands-poised' approach. Midwives, in particular those who had been qualified longer, felt that the 'hands-on' requirement of the care bundle meant that practice had gone 'full circle' and a return to previous practice. Instead of finding this frustrating, however, this was treated in a humorous way:

There were a generation that were hands-off/handspoised, which I always found really weird, but I was a hands-on, that's how I was taught. (Midwife)

This theme was not unique to practices relating to hand position at the time of birth. It also emerged with relation to midwives performing episiotomies and to instrument choices for operative vaginal births. In terms of episiotomy it was felt that the pendulum had swung from midwives performing these for every primiparous woman, to only performing a few and this had created deskilling. There was unanimous agreement that neither extreme was correct practice, but there was a feeling that there should be a middle ground:

They [episiotomies] were in fashion and every firsttime mum had an episiotomy whether she needed one or not. So probably that's when I was trained.... then we went through a stage where nobody was having an episiotomy-how can you teach anybody to do them? (Midwife)

In terms of instrument choice, while this was outside the scope of the care bundle, many participants talked about the shift towards the 'heavy use' of forceps and felt that this should be addressed:

People pick the forceps culturally...there's such an element of fear amongst junior doctors of failing to do something in the room. The other thing that they 
get told off for is using two instruments, so if they use ventouse and they fail... (Obstetrician)

\section{Theme 4: Perceptions of what women want}

This theme reflected a number of factors expressed as reservations for using the care bundle, which related to clinicians' values and perceptions of what women wanted. There were three subthemes: (1) philosophy of care, (2) personal values, and (3) provision of information to women.

\section{Philosophy of care}

Participants, notably midwives, reported that the care bundle changed the current model of care and increased interventions. Midwives, as facilitators of vaginal birth, expressed some reluctance to use procedures which they felt caused overmedicalisation. Some participants felt that births requiring minimal intervention and medicalisation were to be regarded as an achievement. Any interventions took away this feeling of triumph, though this achievement appeared to relate to that of the clinician, not of outcome for the woman:

I haven't had a third or fourth degree tear, touchwood....the intact perineum that's a bit of an achievement....And then it's almost like we're doing a PR, but that's no reward. (Midwife)

While others agreed that this was an intervention, it was felt good communication was key to using all elements consistently:

It's quite a big intervention, isn't it, to touch the woman there....The woman we had this morning who had a pool birth, she had a lovely birth, lovely intact perineum, and when we were inspecting the perineum afterwards we said about the new guidance to do with PR and she had heard of it....so she consented. (Midwife)

\section{Personal values}

Often participants expressed some anxiety about using some elements of the care bundle, when they themselves would not like it as part of their care:

I've been doing it [PR check], because it's part of the study, but I don't like it. And if I was giving birth and I had an intact perineum, I don't think I'd particularly want somebody doing a PR on me. (Midwife)

This feeling of not liking doing something was particularly prominent in midwives reporting of performing episiotomies. As indicated in a previous theme, there was universal agreement that there was deskilling around midwives performing episiotomies. This lack of confidence was sometimes driven by personal fear, "historically, midwives don't like doing them, do we...I just don't like that sound' (midwife), and sometimes driven by women's fear, which in term affected a midwife's confidence:
Lots of people will say, 'I don't want to be cut, I'll just tear, if that's okay.' It's individual. At which point, it puts the whole fear of whether you can go through with that. (Midwife)

\section{Provision of information to women}

There was a range in opinions as to whether the information sheet for women about the project was appropriate. Some felt that women receive too much information when they are pregnant and that they either do not read it or do not take it on board. Some felt the information was 'too scary' or 'too explicit'. However, most participants expressed their belief that women liked the information sheet; it had encouraged clinicians to talk about perineal trauma and educating women was a positive step:

People feel really angry that actually they have no idea that these sorts of things could happen. This project can only really be a good thing in terms of educating them [women] on what can be a normal part of a vaginal delivery. I think that's going to be really positive for everybody. (Obstetrician)

\section{DISCUSSION}

This paper presents clinicians' perspectives of the OASI Care Bundle, implemented as part of a QI programme with national reach and a complex evaluation attached to it. Our findings suggest that there was a mixed reaction by both obstetricians and midwives towards acceptance of the care bundle. To our knowledge, this study offers unique insights as previous studies to reduce severe perineal trauma have focused on the effectiveness of interventions ${ }^{6101125}$ but have not reported in detail clinicians' attitudes towards these interventions. The importance of such qualitative insight within maternity was recently highlighted $^{26}$ as it has implications for implementation, adoption and fidelity of an intervention. Reflection of the identified themes allows an opportunity to raise awareness of potential barriers and enablers which would be considered before implementing similar initiatives.

\section{Theme 1: Implementation strategies}

Adoption, or failure of adoption, of a new intervention is reliant on many components. This QI project was designed with the implementation strategy given as much consideration as the intervention itself. Despite best efforts, it is likely that application of the implementation strategy varied by units which may have impacted on adoption and acceptability of the intervention-notably the issues voiced around the introduction of the intervention. The launch event had the potential to create engagement within the unit. Our findings also highlight the importance of a targeted communication strategy when starting an initiative. The project had a staggered roll-out, and with this came increased communications about the OASI Care Bundle over time. It is possible that the opinions of clinicians in the later regions were affected by this; 
however, even in region 4 there were mixed reactions to the bundle.

\section{Theme 2: Opportunities to use the OASI Care Bundle}

The observation that mentors found it challenging to practise the MPP technique because their students attended births is an important issue to consider when introducing an intervention as efforts need to be made to ensure that all clinicians get sufficient opportunity to gain and become confident users of new skills. This theme also captured the importance of time when implementing a new initiative. The OASI Care Bundle necessitated both midwives and obstetricians to change their behaviour, in terms of learning and using standardised techniques for second-stage perineal management and a change (for some) in the way that they informed women about the risks of perineal trauma. It is important not to underestimate the time that it takes for an intervention to be adopted.

\section{Theme 3: Does practice need to change?}

Clinicians in our study placed high value on their ability to use clinical judgement and their personal values are an almost unavoidable influence when providing care. Recent studies have shown that this may vary depending on the nature and framing of the issue. ${ }^{27}$ This ought to be a careful consideration for any QI intervention that requires behaviour change.

Midwives were more likely to discuss what we termed the 'ebb and flow' of maternity practices, particularly the 'hands-on' or 'hands-poised' approaches for perineal protection and this reflects the often conflicting findings of studies. ${ }^{6} 102428$ Both midwives and obstetricians reported similar views regarding the need for current practice to change, in particular the evidence supporting the elements of the OASI Care Bundle. There were some participants from both professional groups reporting reluctance to perform PR checks as they did not consider they were indicated. However, undiagnosed, or 'missed' OASI is a breach of duty and potential cause for litigation. ${ }^{29}$ In response to these concerns the project developed some 'frequently asked questions' (https://www. rcog.org.uk/en/guidelines-research-services/auditquality-improvement/oasi-care-bundle/oasi-faqs/).

\section{Theme 4: Perceptions of what women want}

Midwives were more likely to report themes alluding to 'Perceptions of what women want', especially the subthemes of 'Philosophy of care' and 'Personal values'. The ability to understand women's needs is an important midwifery skill and research has found that women give midwives authority to make decisions, perceiving them 'to know best'. ${ }^{30}$ However, there were some suggestions that some clinicians do not fully understand the balance between discussing risk and maintaining women's autonomy. ${ }^{31}$ This is an important balance, as women have the right to make informed and autonomous choices and indeed England's Better Births policy initiatives (as well as similar initiatives in Wales and Scotland) aim for women to receive unbiased information, enabling them to develop a personalised maternity care plan based on their decisions and not that of healthcare professionals. ${ }^{32} 33$

Adoption of the care bundle within a unit was dependent on its acceptability to both service users and service providers, alongside institutional support. Failure to adopt new practices is commonly reported in the improvement literature, even when there is substantial evidence of potential benefits to patients and the health system. ${ }^{3435}$ In synthesising these themes and patterns in the data set, we propose that early adoption of the OASI Care Bundle was associated with a number of cognitive, interpersonal and organisational factors. For instance, although the intervention had prominent central support, implementation within each unit was subject to local organisational factors, particularly with the introduction of the intervention and provision of training. Cognitive and interpersonal factors that we identified such as personal values, unit-interprofessional and unit-level awareness have similarly been noted in other maternity interventions and health psychology has been applied to offer theories of behaviour which explain enablers and barriers to uptake. ${ }^{36-38}$

Other reasons for any failure to adopt the intervention may be because some clinicians, as autonomous practitioners, may not value the intervention as they might have felt that other intrapartum perineal techniques, such as warm compresses, should have been incorporated into the OASI Care Bundle. Due to the quality of the evidence, ${ }^{39}$ there was much discussion about warm compresses during development of the OASI Project; however, due to the wide variation in practice (whether the compress is held continuously, what is used for the compress, the temperature, when it is reheated and ability to have a facility for heating compresses) it was decided that the clinical practicalities of ensuring standardisation made it unfeasible to include as a component of the care bundle. Use of compresses was encouraged as part of intrapartum care, but their omission from the care bundle may have caused some resistance. It is clear from the findings here that gaining buy-in for an intervention is an important implementation tool. Other areas to consider are the current strains on maternity services. Pressures such as staffing level and increased complexity of births are well documented within the UK. ${ }^{40}$ These issues did not emerge as a theme within our data; however, it is possible that they were underlying factors which could be additional barriers to adoption of a new intervention.

Training gaps were dominant throughout-for obstetricians this focused on instrument choice and episiotomy technique. For midwives this centred around when and how to perform episiotomies and secondstage perineal care. Episiotomies can be a contentious issue and in the National Health Service a restricted approach to performing them has been adopted, which has created a practice gap among more junior staff. ${ }^{41}$ Midwife inexperience with this procedure has been 
found in other studies, ${ }^{42}$ suggesting that this knowledge gap is widespread and requires continual professional development.

This study has limitations. While FGs produce data through social interaction and the dynamic interaction can stimulate thoughts they can also inhibit participants from divulging their true opinions, in a manner that does not impact one-on-one interviews. All the FGs were held in the participants' place of work and while they were on shift, which affected who was able to attend and for how long. This on occasion meant that discussions felt somewhat rushed (ie, they could have lasted longer) and participants were called away to emergency situations. Some of the FGs were attended by a smaller or larger (10) number of participants than ideally prescribed (6). This was due to clinical pressures and unpredictability of people being available on the day as well as the commitment of doing the FG on a single site visit to ensure it was feasible to conduct them all. As the FGs took place over a period of time, we did not have any control on whether participants may have met with colleagues across units and discussed their views of the OASI Care Bundle-although this per se is not a methodological limitation, as our qualitative approach aimed at eliciting participants' views regardless of how these views were formulated. Additionally, the sampling framework for the study meant that all participants were volunteers which is open to self-selection bias. The majority of participants were women under the age of 40. Men and older women might have had different opinions. Lastly, this study only covers data from frontline providers; further data are required to assess implementability and scalability of the OASI Care Bundle from senior and service managers, as well as women using these services.

The study also has strengths. The sampling framework covered a large number of units with different characteristics and varied clinical contexts across the UK. We were able to reach 101 participants from a range of experience levels who were encouraged to speak freely which allowed for a wide range of perspectives of the OASI Care Bundle and data saturation was reached with the emergence of no new themes. Findings from this study are therefore likely to be relevant to other maternity units, both in the UK and globally, which may consider implementing this care bundle. Lastly, the FGs were all facilitated by the same trained clinical researcher, which enhances the consistency of the data collection. The fact that the facilitator (PB) was a clinician would be expected to have had an impact on the nature of the discussion around the OASI Care Bundle: we consider it a strength of the study as it facilitated trust in a colleague with experience of the front line of a unit and genuine expression of views, including concerns. We do acknowledge that a researcher of different profile (eg, scientist) might have elicited a somewhat different pattern of responses.

\section{CONCLUSION}

This study found that adoption of the OASI Care Bundle across 16 UK units was influenced by four main factors: (1) the way in which the intervention was introduced and implemented in units, (2) opportunities to use the OASI Care Bundle, (3) how receptive participants were to changing their practice, and (4) personal perceptions of what women want. Our synthesis suggests that cognitive and interpersonal factors at the level of individual providers as well as organisational factors at the level of the unit and the central OASI programme team underlined the above and determined the level of success of implementation and adoption of the bundle across studied units. The above findings have informed the development of OASI2, which will be introduced in 2020 in order to scale up and sustain uptake of the OASI Care Bundle (https://www.health.org.uk/funding-andpartnerships/programmes/oasi2-care-bundle). These insights can further be used to introduce other QI initiatives within maternity, and also other healthcare settings.

\section{Author affiliations}

${ }^{1}$ Royal College of Obstetricians and Gynaecologists, London, UK

${ }^{2}$ Croydon University Hospital, Croydon, UK

${ }^{3}$ Health Services Research and Policy, London School of Hygiene and Tropical Medicine, London, UK

${ }^{4}$ Centre for Nursing, Midwifery and Allied Health Professional Research, University College London Hospitals NHS Foundation Trust, London, UK

${ }^{5}$ Royal College of Midwives, London, UK

${ }^{6}$ University Hospitals Plymouth NHS Trust, Plymouth, UK

${ }^{7}$ Centre for Implementation Science, King's College London, London, UK

Acknowledgements We thank all the clinicians who gave up their time to take part in the study. We hugely appreciate their engagement and openness. We thank the obstetric and midwifery champions at each of the 16 participating units for their hard work and dedication to implementing all aspects of this QI project. The OASI Care Bundle team acknowledge the support of the Health Foundation.

Contributors RT, IGU, LS, RF and NS conceptualised the OASI Care Bundle. PB, IGU and NS led on the evaluation of the study. RT, LS, AH and VN led on the implementation of the study. PB organised and facilitated the focus groups, performed data management and analysis, and drafted the manuscript. JMH was an independent second coder for data analysis, reviewing the codes and themes in order to develop new insights and minimise bias. All authors (PB, RT, IGU, JMH, AH, $\mathrm{RF}, \mathrm{EM}, \mathrm{VN}$ and NS) critically reviewed the manuscript and provided input before publication. NS provided significant overall guidance.

Funding The OASI Project is part of the Health Foundation's Scaling-Up Improvement programme (award number 7674). The Health Foundation fully funded both the implementation and the evaluation of the project.NS' research is supported by the National Institute for Health Research (NIHR) Applied Research Collaboration (ARC) South London at King's College Hospital NHS Foundation Trust. NS is a member of King's Improvement Science, which offers cofunding to the NIHR ARC South London and comprises a specialist team of improvement scientists and senior researchers based at King's College London. Its work is funded by King's Health Partners (Guy's and St Thomas' NHS Foundation Trust, King's College Hospital NHS Foundation Trust, King's College London, and South London and Maudsley NHS Foundation Trust), Guy's and St Thomas' Charity and the Maudsley Charity.

Disclaimer The views expressed are those of the authors and not necessarily those of the Health Foundation, the NIHR, the National Health Service (NHS) or the Department of Health and Social Care.

Competing interests NS is the director of London Safety and Training Solutions, which provides patient safety advisory and training services on a consultancy basis to hospitals in the UK and internationally. EM is a trustee of the RCOG. 
Patient and public involvement Patients and/or the public were involved in the design, or conduct, or reporting, or dissemination plans of this research. Refer to the Methods section for further details.

\section{Patient consent for publication Not required.}

Ethics approval This QI project was reviewed by NHS Health Research Authority in October 2016 and approved as a service evaluation (Ref 60/86/81). We thus sought and obtained relevant local approvals, including from the Caldicott Guardians across all the NHS trusts involved in this evaluation. Only named members of the Project Team have access to the data. They have completed the mandatory Information Governance Training and are aware of the requirements of confidentiality and understand that any breach will be reported.The RCOG and LSHTM are registered under the Data Protection Act and are fully compliant with the NHS Information Governance Toolkit for Hosted Secondary Use Teams. Participants (clinicians) who took part in qualitative research activities all provided their verbal consent to do so and were made aware that they could withdraw this at any time.

Provenance and peer review Not commissioned; externally peer reviewed.

Data availability statement № data are available. No additional data available.

Open access This is an open access article distributed in accordance with the Creative Commons Attribution Non Commercial (CC BY-NC 4.0) license, which permits others to distribute, remix, adapt, build upon this work non-commercially, and license their derivative works on different terms, provided the original work is properly cited, appropriate credit is given, any changes made indicated, and the use is non-commercial. See: http://creativecommons.org/licenses/by-nc/4.0/.

\section{ORCID iDs}

Posy Bidwell http://orcid.org/0000-0001-8506-9880

Ipek Gurol-Urganci http://orcid.org/0000-0002-6517-3485

Nick Sevdalis http://orcid.org/0000-0001-7560-8924

\section{REFERENCES}

1 LaCross A, Groff M, Smaldone A. Obstetric anal sphincter injury and anal incontinence following vaginal birth: a systematic review and meta-analysis. J Midwifery Womens Health 2015;60:37-47.

2 Priddis HS. Autoethnography and severe perineal trauma--an unexpected journey from disembodiment to embodiment. BMC Womens Health 2015;15:88.

3 Evans C, Archer R, Forrest A, et al. Management of obstetric anal sphincter injuries (OASIS) in subsequent pregnancy. $J$ Obstet Gynaecol 2014;34:486-8.

4 NHS Litigation Authority. Maternity claims: information sheet 13 perineal trauma. Ten years of maternity claims an analysis of NHS litigation authority data. London: National Health Service (NHS), 2012.

5 McLeod NL, Gilmour DT, Joseph KS, et al. Trends in major risk factors for anal sphincter lacerations: a 10-year study. J Obstet Gynaecol Can 2003;25:586-93.

6 Ismail KMK, Paschetta E, Papoutsis D, et al. Perineal support and risk of obstetric anal sphincter injuries: a Delphi survey. Acta Obstet Gynecol Scand 2015;94:165-74.

7 Trochez R, Waterfield M, Freeman RM. Hands on or hands off the perineum: a survey of care of the perineum in labour (HOOPS). Int Urogynecol J 2011;22:1279-85.

8 Andrews V, Sultan AH, Thakar R, et al. Risk factors for obstetric anal sphincter injury: a prospective study. Birth 2006;33:117-22.

9 Naidu M, Kapoor DS, Evans S, et al. Cutting an episiotomy at 60 degrees: how good are we? Int Urogynecol J 2015;26:813-6.

10 Laine K, Skjeldestad FE, Sandvik L, et al. Incidence of obstetric anal sphincter injuries after training to protect the perineum: cohort study. BMJ Open 2012;2:e001649.

11 Rasmussen OB, Yding A, Anh $\varnothing \mathrm{J}$, et al. Reducing the incidence of obstetric sphincter injuries using a hands-on technique: an interventional quality improvement project. BMJ Qual Improv Rep 2016;5:u217936.w7106.

12 Leenskjold S, Høj L, Pirhonen J. Manual protection of the perineum reduces the risk of obstetric anal sphincter ruptures. Dan Med J 2015;62.

13 Naidu M, Sultan AH, Thakar R. Reducing obstetric anal sphincter injuries using perineal support: our preliminary experience. Int Urogynecol J 2017;28:381-9.

14 Basu M, Smith D, Edwards R, et al. Can the incidence of obstetric anal sphincter injury be reduced? the STOMP experience. Eur J Obstet Gynecol Reprod Biol 2016;202:55-9.

15 Gurol-Urganci I, Cromwell DA, Edozien LC, et al. Third- and fourth-degree perineal tears among primiparous women in England between 2000 and 2012: time trends and risk factors. BJOG 2013;120:1516-25

16 Ampt AJ, Patterson JA, Roberts CL, et al. Obstetric anal sphincter injury rates among primiparous women with different modes of vaginal delivery. Int J Gynaecol Obstet 2015;131:260-4.

17 Tung CW, Cheon WC, Tong WMA, et al. Incidence and risk factors of obstetric anal sphincter injuries after various modes of vaginal deliveries in Chinese women. Chin Med J 2015;128:2420-5.

18 Bidwell P, Thakar R, Sevdalis N, et al. A multi-centre quality improvement project to reduce the incidence of obstetric ana sphincter injury (OASI): study protocol. BMC Pregnancy Childbirth 2018;18:331.

19 Gurol-Urganci I, Bidwell P, Sevdalis N. Impact of a quality improvement project to reduce the rate of obstetric anal sphnicter injury: a multi-centre study with a stepped-wedge design. BJOG 2018.

20 Wellings K, Branigan P, Mitchell K. Discomfort, discord and discontinuity as data: using focus groups to research sensitive topics. Cult Health Sex 2000;2:255-67.

21 Pope C, Ziebland S, Mays N. Analysing qualitative data. BMJ 2000;320:114-6.

22 Fereday J, Muir-Cochrane E. Demonstrating rigor using thematic analysis: a hybrid approach of inductive and deductive coding and theme development. Int J Qual Methods 2006;5:80-92.

23 O'Brien BC, Harris IB, Beckman TJ, et al. Standards for reporting qualitative research: a synthesis of recommendations. Acad Med 2014;89:1245-51.

24 McCandlish $\mathrm{R}$, Bowler U, van Asten $\mathrm{H}$, et al. A randomised controlled trial of care of the perineum during second stage of normal labour. $\mathrm{Br}$ J Obstet Gynaecol 1998;105:1262-72.

25 De Meutter L, D van Heesewijk A, van der Woerdt-Eltink I, et al. Implementation of a perineal support programme for reduction of the incidence of obstetric anal sphincter injuries and the effect of noncompliance. Eur J Obstet Gynecol Reprod Biol 2018;230:119-23.

26 Denny E, Weckesser A. Qualitative research: what it is and what it is not. BJOG 2018;126.

27 Hermann $\mathrm{H}$, Trachsel M, Biller-Andorno N. Physicians' personal values in determining medical decision-making capacity: a survey study. J Med Ethics 2015;41:739-44.

28 Bulchandani S, Watts E, Sucharitha A, et al. Manual perineal support at the time of childbirth: a systematic review and meta-analysis. BJOG 2015;122:1157-65.

29 Sultan A, Ritchie A, Mooney G. Obstetric anal sphnicter injuries: review of recent medio-legal aspects. J Patient Saf Risk Manag 2016;22:57-60.

30 Bluff R, Holloway I. 'They know best': women's perceptions of midwifery care during labour and childbirth. Midwifery 1994;10:157-64.

31 Murphy M. Maternal autonomy. Br J Midwifery 2016;24:371-3.

32 Kruske S, Young K, Jenkinson B, et al. Maternity care providers' perceptions of women's autonomy and the law. BMC Pregnancy and Childbirth2013;13.

33 Review NM. Better births: improving outcomes of maternity services in England. A five year plan forward view for maternity care, 2016: 1-124.

34 Edmondson A. Psychological safety and learning behavior in work teams. Adm Sci Q 1999;44:350-83.

35 Kimberly JR, Evanisko MJ. Organizational innovation: the influence of individual, organizational, and contextual factors on hospital adoption of technological and administrative innovations. Acad Manage J 1981;24:689-713.

36 Flannery C, McHugh S, Anaba AE, et al. Enablers and barriers to physical activity in overweight and obese pregnant women: an analysis informed by the theoretical domains framework and COM-B model. BMC Pregnancy Childbirth 2018;18:178.

37 Jeffery J, Hewison A, Goodwin L, et al. Midwives' experiences of performing maternal observations and escalating concerns: a focus group study. BMC Pregnancy Childbirth 2017;17:282.

38 Lundgren I, Healy $\mathrm{P}$, Carroll $\mathrm{M}$, et al. Clinicians' views of factors of importance for improving the rate of VBAC (vaginal birth after caesarean section): a study from countries with low VBAC rates. BMC Pregnancy Childbirth 2016;16:350.

39 Aasheim V, Nilsen ABV, Reinar LM, et al. Perineal techniques during the second stage of labour for reducing perineal trauma. Cochrane Database Syst Rev 2017;6:CD006672.

40 Sandall J, Homer C, Sadler E. Staffing in maternity units: getting the right people in the right place at the right time: the kings fund, 2011.

41 Tincello DG, Williams A, Fowler GE, et al. Differences in episiotomy technique between midwives and doctors. BJOG 2003;110:1041-4.

42 Crowe G, Miles M, Nagle C. Exploring midwives' practice and experience of episiotomy. Women and Birth 2018;31:S49. 